\title{
Versatile Applications of Heterocyclic Compounds: Special Attention to Nanomaterials in Cancer Therapy
}

\section{Jagadevappa Patil}

VT's Shivajirao S Jondhle College of Pharmacy, Asangaon-421 601, Shahapur, Thane, Maharashtra, India

*Corresponding author: Jagadevappa Patil, VT's Shivajirao S Jondhle College of Pharmacy, Asangaon-412 601, Thane, Maharashtra, India, Tel: +91 9448816812; Email: pharmajspatil@gmail.com

Received date: November 15, 2016; Accepted date: November 21, 2016; Published date: November 25, 2016

Copyright: (c) 2016 Patil J. This is an open-access article distributed under the terms of the Creative Commons Attribution License, which permits unrestricted use, distribution, and reproduction in any medium, provided the original author and source are credited.

\section{Introduction}

Contribution of medicinal chemistry to the biological, medicinal and pharmaceutical field is gigantic including drug expansion, detection, design and identification of bioactive compounds. Heterocyclic compounds represent vital importance of medicinal chemistry and have paying robust curiosity with a wider scope to synthesize and process various types of pharmacological and biological properties. Nanotechnology is the art of deploying materials on atomic or molecular scales especially to shape nanostructures which are considered to be the suitable tools to solve the problems of health care sectors. Finding a solution to the problem of severe side effects, drug resistance, and poor pharmacokinetic properties of many bioactive compounds is an urgent global health care priority, which could potentially avoid thousands of needless deaths, and reduce the burden on healthcare systems around the world. Nanostructured heterocyclic compounds can show significantly enhanced biological properties with reduced unwanted effects compared with conventional compounds. Synthetic heterocycles have extensive therapeutic uses available in different therapeutic categories such as antibacterial, antifungal, analgesic, anti-inflammatory, anti-mycobacterial, anti-tubercular, antimalarial, trypanocidal, anti-HIV activity, muscle relaxants, anticonvulsant, anti-tumoral, anti-leishmanial agents, genotoxic, herbicidal, anticancer and lipid peroxidation inhibitor, hypnotics, antidepressant, anthelmintic and insecticidal agents [1-7].

\section{Relevance of Heterocyclic Nanostructures in Cancer Therapy}

The technology and reasoning behind drug design are closely interrelated to the pre mediated inclusion of heterocyclic fragments with explicit physicochemical characteristics. Effectiveness and discernment through bio isosteric substitutions, lipophilicity, polarity, and aqueous solubility can eventually be modified to the point of changing and conditioning the possible mechanisms of action of heterocyclic drugs in an effort to attain molecularly targeted therapeutic agents [8]. Regardless of their flexibility and effectiveness, as for any other therapeutics, there are many issues obstructing broader application and auxiliary expansion of such compounds into market drugs. Treatment of cancer is perhaps most widely suffering health care event due to the inherent confines regarding foremost therapeutic routes of chemotherapy, attendant side effects and toxicity to unaffected healthy cells. Such venomous effects may be skirted by selective targeted drug delivery approaches directly into malignant cells [9]. With respect to this, nanostructure based delivery tools are the most attractive solutions associated with various cancer chemotherapy hindrances. In order to circumvent the poor physicochemical characteristics of many therapeutic heterocyclic compounds including their pharmacokinetic and dynamic properties and advance their use towards clinical translation, numerous formulation development studies have been conducted using various nanostructured systems. The nanostructured heterocyclic compounds are capable enough to bind and/or encapsulate with targeting carrier and allows the exploit of improved cell permeability and retaining effect appropriate for malignant targeting. This facilitates for an accumulated concentration of therapeutic agent at the tumor site with a resultant increase in efficiency while decreasing the unwanted side effects and avoids effecting healthy cells. The surface nanocarriers may be conjugated with extra targeting moieties to enhance the selectivity towards malignant tissues with specific overexpressed receptors, and/or other biomarkers [10]. Nanostructured carrier systems include polymeric nanoparticles, liposomes; albumin bound metallic nanoparticles, and dendrimers [11]. Regardless of widespread nanomaterials are in use, encapsulated nanostructures such as polymeric nanoparticles and liposomal formulations are found to be serving most favourable with more than 30 nano formulations in ongoing clinical trials [12].

\section{Conclusion}

Drug targeting strategies through nanostructured systems are found to be most promising with added advantages such as improved cell permeability and retention effect of heterocyclic therapeutics for better biocompatibility. It is also possible to achieve specific drug targeting with negligible side effects. Although there are many published literatures about nanostructured systems for anticancer drugs, few have entered into clinical trials and fewer are approved for human use. Still several trials are needed to address many problems such as an ideal and reproducible physicochemical depiction of nanostructures for an industrialized vectorization of heterocyclic compounds, safety issues in order to achieve a swiftly effectual cancer therapy.

\section{References}

1. Mittal A (2009) Synthetic Nitroimidazoles: Biological activities and mutagenicity relationships. Sci Pharm 77: 497-520.

2. Nagalakshmi G (2008) Synthesis, Antimicrobial and Anti-inflammatory Activity of 2,5-disubstituted-1,3,4-oxadiazoles. Indian J Pharm Sci 70: 49-55.

3. Joule JA, Mills K (2000) Heterocyclic Chemistry ( $4^{\text {th }}$ edn.), Wiley-Blackwell Publishing.

4. Nekrasov DD (2001) Biological Activity of 5- and 6-Membered Azaheterocycles and Their Synthesis from 5-Aryl-2, 3-Dihydrofuran-2,3diones. Chem Hetero Comp 37: 263-275.

5. Sperry JB, Wright DL (2005) Furans, thiophenes and related heterocycles in drug discovery. Curr Opin Drug Discov Devel 8: 723-740. 
Citation: Patil J (2016) Versatile Applications of Heterocyclic Compounds: Special Attention to Nanomaterials in Cancer Therapy. J Pharmacovigil 4: e164. doi:10.4172/2329-6887.1000e164

Page 2 of 2

6. Polshettiwar V, Varma RS (2008) Greener and expeditious synthesis of bioactive heterocycles using microwave irradiation. Pure Appl Chem 80: 777-790.

7. Katritzky AR (1992) Heterocyclic Chemistry: An Academic Subject of Immense Industrial Importance. Chemi Heterocycl Comp 28: 241-259.

8. Gomtsyan A (2012) Heterocycles in drugs and drug discovery. Chem Heterocycl Compd 48: 7-10.

9. Peer D, Karp JM, Hong S, Farokhzad OC, Margalit R, et al. (2007) Nanocarriers as an emerging platform for cancer therapy. Nat Nanotechno 2: 751-760.
10. Xu X, Ho W, Zhang X, Bertrand N, Farokhzad O (2015) Cancer nanomedicine: From targeted delivery to combination therapy. Trends $\mathrm{Mol}$ Med 21: 223-232.

11. Sagnella SM, McCarroll JA, Kavallaris M (2014) Drug delivery: Beyond active tumour targeting. Nanomed Nanotechnol Biol Med 10: 1131-1137.

12. Wicki A, Witzigmann D, Balasubramanian V, Huwyler J (2015) Nanomedicine in cancer ther apy: Challenges, opportunities, and clinical applications. J Control Release 200: 138-157. 